This item was submitted to Loughborough's Research Repository by the author.

Items in Figshare are protected by copyright, with all rights reserved, unless otherwise indicated.

\title{
Materials design towards sport textiles with low-friction and moisture-wicking dual functions
}

PLEASE CITE THE PUBLISHED VERSION

http://dx.doi.org/10.1016/j.matdes.2015.08.107

PUBLISHER

(C) Elsevier

VERSION

AM (Accepted Manuscript)

\section{PUBLISHER STATEMENT}

This work is made available according to the conditions of the Creative Commons Attribution-NonCommercialNoDerivatives 4.0 International (CC BY-NC-ND 4.0) licence. Full details of this licence are available at: https://creativecommons.org/licenses/by-nc-nd/4.0/

\section{LICENCE}

CC BY-NC-ND 4.0

\section{REPOSITORY RECORD}

Dong, Yuliang, Junhua Kong, Chenzhong Mu, Chenyang Zhao, Noreen L. Thomas, and Xuehong Lu. 2015. "Materials Design Towards Sport Textiles with Low-friction and Moisture-wicking Dual Functions". figshare. https://hdl.handle.net/2134/19028. 


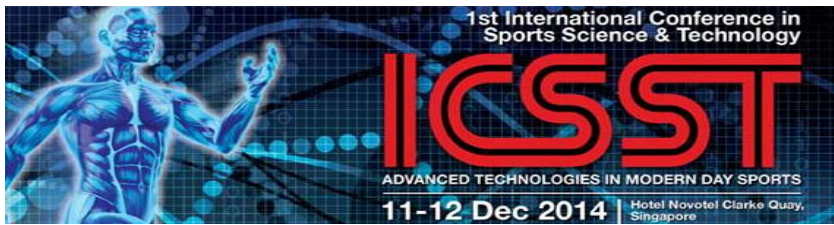

Advanced Materials for

Sports Technology

www.icsst14.com

\title{
Materials Design towards Sport Textiles with Low-Friction and Moisture- Wicking Dual Functions
}

\author{
Yuliang Dong ${ }^{1,2,3}$, Junhua Kong ${ }^{1}$, ChenzhongMu ${ }^{1}$, Chenyang Zhao ${ }^{1}$, Noreen L. Thomas ${ }^{3}$, \\ Xuehong Lu ${ }^{1,2, *}$
}

${ }^{1}$ School of Materials Science and Engineering, Nanyang Technological University, 50 Nanyang Avenue, Singapore 639798

${ }^{2}$ Institute for Sport Research, Nanyang Technological University, 50 NanyangAvenue,Singapore 639798

${ }^{3}$ Department of materials, Loughborough University, Loughborough, Leicestershire, LE11 3TU, United Kingdom

*Corresponding author, E-mail: asxhlu@ntu.edu.sg

\section{Keywords}

Electrospinning, core-shell, textile, low friction, moisture transport

\section{Abstract}

In the field of sportswear, the structure and morphology of textiles are of great importance to achieve good moisture transport and low friction, which are two critical comfort-related properties. To improve these properties, dual-layer nanofibrous nonwoven mats were studied in this work. Core-shell nanofibers with a polyacrylonitrile (PAN)-rich core and a poly(vinylidene fluoride) (PVDF)-rich shell were fabricated by single-spinneret electrospinning and used as the inner layer of 


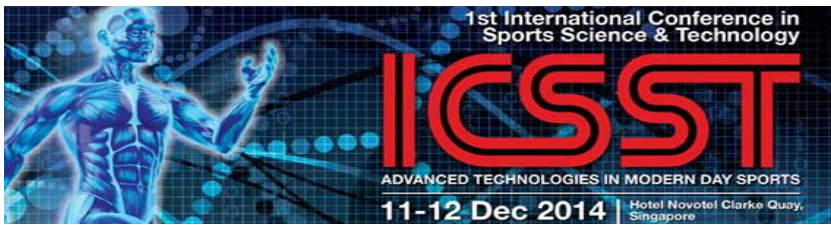

\section{Advanced Materials for Sports Technology \\ www.icsst14.com}

the dual-layer mats, while thick base-treated Cellulose Acetate (CA) nanofibrous mats were used as the outer layer. The core-located PAN and a small amount of PAN on the PAN/PVDF nanofiber surface ensure good moisture transport through the nanofibrous mats. The synergistic combination of a considerably hydrophobic PAN/PVDF inner layer and a highly hydrophilic CA outer layer induces a strong push-pull effect, resulting in efficient moisture transport from the inner to the outer layer. Furthermore, the fluorine-rich PVDF shell of the inner layer touches the human skin and provides a lubricating effect to enhance comfortability. This design provides a promising route for sports textiles with both good moisture-wicking and low friction.

\section{Introduction}

The textile used for sportswear, particularly the layer worn next to the skin, is of great importance for the physiological comfort and sports performance of people. Moisture transport behaviour of the textiles is a key property to consider in design of such textiles. During outdoor activities, especially in hot environments, clothing should allow perspiration to be transported out quickly ${ }^{1}$. Otherwise, body thermal balance cannot be maintained, and as moisture accumulates, the skin would also become more susceptible to abrasive damage and microbial growth ${ }^{2}$.

Another critical comfort-related property for sports textiles is low friction. When a person is playing sports, the inner surface of the sportswear may slide over his/her skin, inducing friction. High friction may cause uncomfortable feeling or even pain of the sportspeople. For instance, the friction between the skin and inner surface of sock fabric can cause friction blisters due to cyclic relative movements. Fluoropolymers have very low coefficients of friction (varying from 0.02 to 0.2 depending on the fluoropolymer type, loading and the sliding speed) and poor adhesion to most 


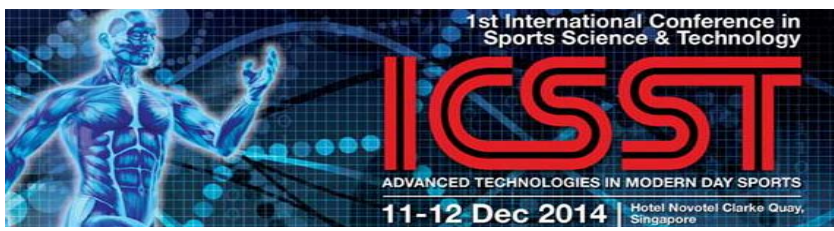

\section{Advanced Materials for Sports Technology \\ www.icsst14.com}

substances because of their low surface energy. ${ }^{3}$ For example, Bertaux ${ }^{4}$ investigated the friction of socks made of different types of fibers, and found that the socks containing polytetrafluoroethylene have lower friction and are more comfortable. Simone Cazzaniga ${ }^{5}$ also pointed out that fluorinesynthetic fiber socks are more comfortable and healthy.

Recently, many studies on fabrication of multifunctional textiles, such as those with special wettability, antibacterial property and anti-UV properties, have been reported. Huang et al. prepared superhydrophobic $\mathrm{TiO}_{2}$-fabric through a one-pot hydrothermal reaction on a cotton fabric for UV shielding, self-cleaning and oil-water separation. ${ }^{6} \mathrm{Ag}$ NPs were also synthesized on fabric surface to achieve good color fastness, antimicrobial property and UV protection. ${ }^{7}$ Yu et al. found that multifunctional metal commingled yarns composed of antibacterial nylon/polyester/stainless steel wires could achieve good moisture wicking and antibacterial properties. ${ }^{8}$ Modified cotton fabrics with water- and oil-repellent, antibacterial and flame-retardant properties have also been reported. $^{9}$ However, so far there is no report on multifunctional textiles that exhibit both lowfriction and good moisture transport properties, although both are important for the comfortability of sports textiles.

Dual-layer textiles are candidates to combine good moisture transport and low friction together in the same material. Typical dual-layer moisture-wicking fabrics have a hydrophobic layer, such as a polypropylene (PP) layer, in direct contact with the skin and a hydrophilic outer layer, which is frequently made of cotton or cotton blends. ${ }^{10}$ The inner layer has low water absorption ability, pushing sweat to the outer layer via capillary motion. The outer layer has high water absorption ability, pulling the sweat out from the inner layer. The push-pull effect facilitates moisture transport and evaporation to the surrounding environment. Fluoropolymers are highly hydrophobic, and 


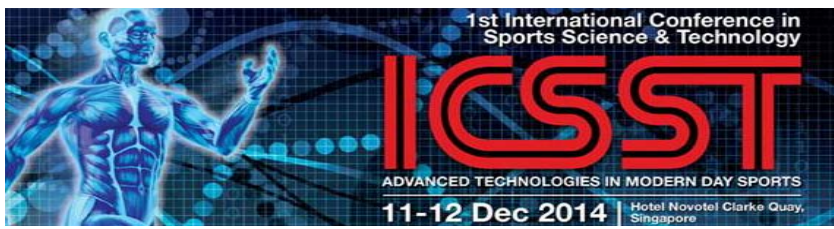

\section{Advanced Materials for Sports Technology \\ www.icsst14.com}

hence the introduction of a fluoropolymer to the inner layer is a possible way of fabricating a duallayer moisture-wicking textile with low friction.

Electrospinning is a popular technique used to produce non-woven mats of polymer nanofibers. ${ }^{11-13}$ The controllability of the thickness of the mats and the size of the nanofibers ${ }^{14}$, as well as the feasibility of facile post-treatment, make electrospun nonwoven mats a promising candidate for further modification of fabrics and introduction of new functionality to the fabrics. For example, in a previous publication ${ }^{15}$, the current authors showed that electrospinning can be used to produce dual-layer nanofibrous mats consisting of a thin inner layer of polystyrene (PS) nanofibers, which have very low water absorption capability, and a thick outer layer of polyacrylonitrile(PAN) nanofibers, which have some hydrophilic groups on surface to facilitate capillary action. The porosity of the PS nanofibers was controlled and also the surface hydrophilicity was tailored by coating with thin layers of polydopamine. In this way it was demonstrated that moisture-wicking could be significantly enhanced.

In this study, core-shell nanofibers with a PAN-rich core and a poly(vinylidene fluoride) (PVDF)rich shell were fabricated by single-spinneret electrospinning and used as the inner layer of duallayer mats, while thick base-treated cellulose acetate (CA) nanofibrous mats, which are hydrophilic, were used as the outer layer. The motivation to use a PAN/PVDF core/shell nanofbrous mat as the textile inner layer that touches the human skin is to achieve low friction with the skin without sacrificing moisture transport property. The core/shell nanofibers have a large amount of fluorine atoms on their surface, making the inner layer fairly hydrophobic and not sticky in wet state as well as inducing lubricating effect to ensure low friction. The major driving force for the moisture transport through the dual-layer nanofibrous mats is the large hydrophobicity 


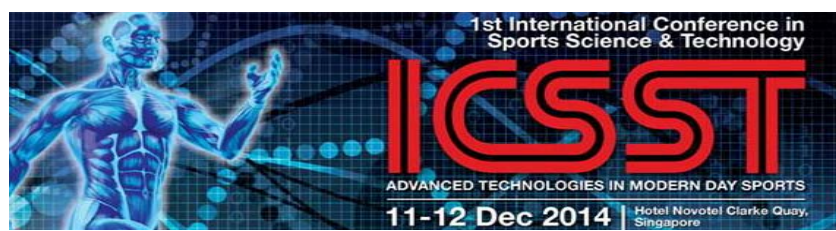

Advanced Materials for

Sports Technology

www.icsst14.com

difference between the inner and outer layers, while the core-located PAN and a small amount of PAN on the core-shell PAN/PVDF nanofiber surface could facilitate the moisture transport from the inner surface to the outer surface; without the core-located PAN and surface-located PAN groups, the moisture would be difficult to pass through a neat PVDF inner layer that has very poor wettability. In this paper, the structures and morphologies of the core-shell nanofibers and dual layer mats are presented, and the effectiveness of our design in providing both low friction and good moisture transport properties is demonstrated.

\section{Experimental Work}

\subsection{Materials}

Polyacrylonitrile $(\mathrm{PAN}, \mathrm{Mw}=150,000)$ was purchased from Aldrich Chemical Company Inc (USA). N, N-Dimethylformamide (DMF) AND ACETONE were supplied by Tedia Company Inc (USA). Poly(vinylidene fluoride-hexafluoropropylene) (P(VDF-HFP), solef 11008) and Cellulose Acetate (CA, Mn 50,000) were purchased from Sigma-Aldrich Chemcals (USA). All materials were used without further purification. For simplicity, $\mathrm{P}(\mathrm{VDF}-\mathrm{HFP})$ is referred as PVDF in this paper.

\subsection{Preparation of nanofibrous mats}

PAN solution in DMF (8 wt\% PAN),PVDF solution in DMF(15 wt\% PVDF), PAN/PVDF solution in DMF(5 wt \% PAN-5 wt \% PVDF, 6wt $\%$ PAN-2wt $\%$ PVDF and 6wt $\%$ PAN- $1 w t \%$ PVDF) and CA solution in DMF/Acetone(13wt\% CA, DMF/Acetone-1/1 by weight) were prepared by 


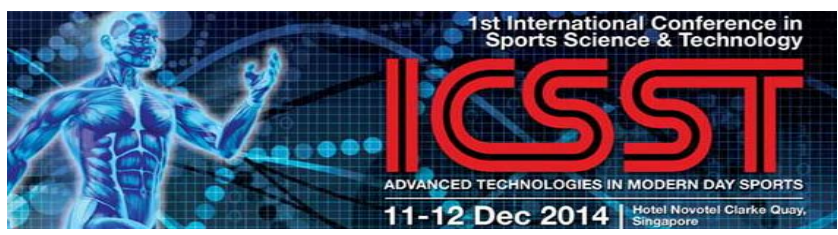

\section{Advanced Materials for Sports Technology \\ www.icsst14.com}

dissolving PAN, PVDF or CA powder in the corresponding solvent at $60{ }^{\circ} \mathrm{C}$ under continuous magnetic stirring. The electrospinning setup and conditions used were the same as those reported in [11]. The spinning time for PAN/DMF, PVDF/DMF and PAN/PVDF/DMF solutions was 25min, whereas CA solutions were spun for 12 hours. After electrospinning, the CA mat was soaked in $0.05 \mathrm{M} \mathrm{NaOH}$ aqueous solution for 16 hours and rinsed with de-ionized water to convert $\mathrm{CA}$ into a cellulose mat. Base-treated CA-PAN/PVDF dual-layer mats were prepared by electrospinning of PAN/PVDF/DMF (5 wt \% PAN-5 wt\% PVDF) solution for 25 min onto base-treated CA mats. To facilitate the discussion below, the dual layer is denoted as D-CA-PAN/PVDF. All the mats were vacuum dried at $60{ }^{\circ} \mathrm{C}$ for $24 \mathrm{~h}$ and then went through a paper laminator ( $\mathrm{PRO} \mathrm{BiO} 320$ ) at $70^{\circ} \mathrm{C}$ and a rolling speed of $50 \mathrm{rpm}$. Solution-cast solid PVDF, PAN,PVDF/PAN blend and CA films were also prepared. The films were cast from the corresponding solutions and dried in a vacuum oven at $80^{\circ} \mathrm{C}$ for $24 \mathrm{hrs}$. CA films were soaked in $0.05 \mathrm{M} \mathrm{NaOH}$ aqueous solution for $4,8,12,16$, 20, 24 hours and rinsed with de-ionized water to convert CA into cellulose to different extents.

\subsection{Characterization}

Morphologies of the mats were examined using field-emission scanning electron microscopy (FESEM) and transmission electron microscopy (TEM). The instruments and experimental conditions used were the same as those reported in [15].The composition of the blend nanofibers was studied by XPS using a VG ESCALAB 220I-XL XPS system (Thermo VG Scientific Ltd., UK). The base pressure was $10^{-9}$ Torr and the X-ray radiation was $\mathrm{Mg}-\mathrm{K} \alpha$ radiation $(1253.6 \mathrm{eV})$ at a power of 200 watts. Fourier Transform Infra-red(FTIR) spectroscopy was recorded using a PerkinElmer (Frontier) spectrometer. Apparent contact angles and moisture transport behaviour of the samples were also measured using the same instruments and experimental conditions as those 


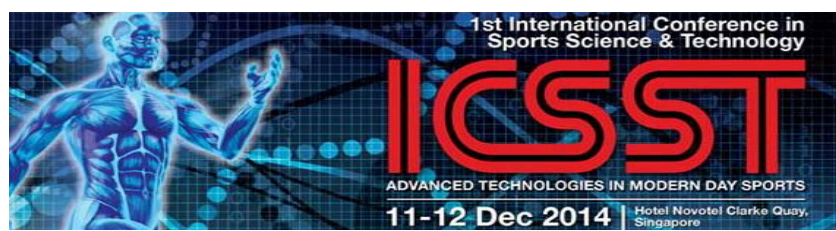

\section{Advanced Materials for \\ Sports Technology \\ $\underline{\text { www.icsst14.com }}$}

reported in [15]. To test the moisture transport behaviors of the nanofibrous mats, a piece of dry mat was placed in the chamber of a moisture management tester (MMT, SDLATLAS), and then a droplet of $0.15 \mathrm{M}$ sodium chloride aqueous solution was sprayed on the top (inner) surface of the mat. Using the MMT, the in-situ water contents of both top (inner) surface and bottom (outer) surface of the mat were measured, and these relative water content values were used to infer the water transport behavior of the sample.[15]

The friction coefficient of the nanofibrous mats was tested using a Tribometer (CSM Instruments). During the test, a Polydimethylsiloxane (PDMS) film was used to simulate the skin and was slid over the surface of the nanofibrous mats and cotton fabric.

In order to test the water absorption capability, cast films were immersed in de-ionized water for 48hours. The films were all of rectangular shape with an area of $20 \mathrm{~cm}^{2}$. Their weights were measured before and after being soaked in the de-ionized water. Water absorption is calculated as the ratio of the weight gained to the original weight.

\section{Results and Discussion}

\subsection{PAN-rich core/ PVDF-rich shell blend nanofibers as the inner layer}

In our design, the electrospun inner layer of the dual-layer structure that is next to the skin should be hydrophobic to trigger a push-pull effect enhancing water transport as well as having low friction with the skin. As a common hydrophobic fluoropolymer, an electrospun PVDF nanofibrous mat is expected to have low friction and could trigger push-pull effects with the hydrophilic outer layer. However, water can hardly pass through the PVDF layer due to its poor wettability. Thus 


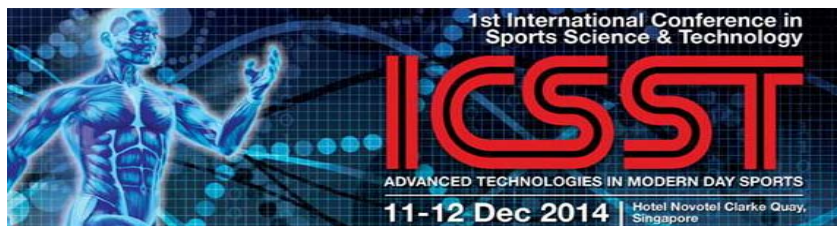

\section{Advanced Materials for Sports Technology \\ $\underline{\text { www.icsst14.com }}$}

PAN was blended with PVDF during the electrospinning process to improve the wettability of the layer.

The morphology of the as-spun PAN-rich core/ PVDF-rich shell blend nanofibers at a PAN to PVDF weight ratio of 1 to 1 were observed by SEM and TEM. The SEM image in Figure1 (a) shows typical morphologies of the as-spun blend nanofibers. The nanofibers are uniform and beadless, and from the image at high magnification, the nanofibers are seen to be solid and rough. (The morphologies of PAN and PVDF nanofibrous mats are shown in FigureS1.) The TEM image in Figure1(b) shows that a core-shell structure is observed within the blend nanofibers, because there is an abrupt change in brightness between the shell and core due to the asymmetric distribution of elements, indicating phase separation during electrospinning. This is because the surface energy of PVDF is lower than that of PAN so that PVDF prefers to stay at the surface of the nanofiber, forming the shell. It worth noting that the distinct core-shell morphology was achieved without incorporation of any salts, which is probably because the solvent used was different from that reported in W Lu's work ${ }^{16}$. DMF may evaporate more slowly than DMF/acetone mixtures and hence give more time for phase separation.

In order to further confirm such core-shell morphology, electrospun PAN/PVDF blend nanofibers were also prepared at a PAN to PVDF weight ratio of 3 to 1 and 6 to 1 , and XPS analysis was carried out. The XPS results and the calculated atomic content of elemental fluorine (F) on the surface are shown in Figure2. The composition of the neat PVDF nanofibers was also measured as a reference. By comparing the $\mathrm{F}$ contents, calculated based on the feed compositions, with the measured surface F content, it is clear that the calculated F content based on the feed PAN/PVDF ratio is much lower than the measured one for the blend nanofibers. The XPS results are shown in 


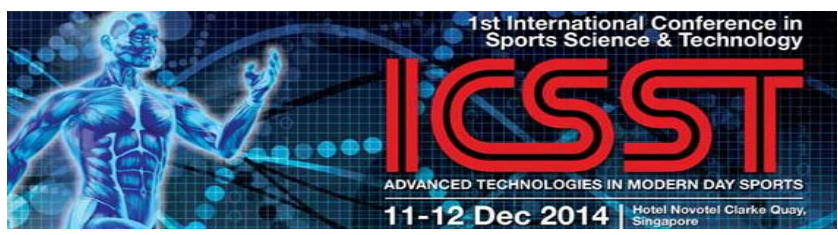

Advanced Materials for

Sports Technology

www.icsst14.com

FigureS2. Since the detection depth of XPS is normally less than $12 \mathrm{~nm}$, and the elemental F only comes from PVDF, it can be concluded that the shell of the blend nanofibers is rich in PVDF. This confirms the PAN-core/PVDF-shell configuration for the PAN/PVDF blend nanofibers. Due to the hydrophobic nature of PVDF, the surface-located PVDF will produce a hydrophobic surface on the blend nanofibers. This is reflected by the similar apparent contact angles of neat PVDF and the blend nanofibers with different ratios (Figure 3).

The moisture management (MMT) results of water transport through the PVDF, PAN and PVDF/PAN blend (PVDF/PAN ratio=1/1) nanofibrous mats are shown in Figures 4 (a), (b) and (c). In these figures, the solid line (green) indicates the water content of the top side of the sample, on which a constant amount of water was sprayed, and the dashed line (blue) indicates the water content of the bottom side of the sample, which is originally dry and gets wet when moisture is transported from the top to bottom surface. ${ }^{17}$ Figure 4 (a) shows the MMT result for PAN nanofibers. Since PAN is hydrophilic, the values of the solid and dashed lines increase from the start and soon reach a steady-state value because water can easily pass through the mat. For pure PVDF, which is shown in Figure 4(b), the value of the dashed (blue) line is kept at zero in the whole testing period, indicating that no water is transported to the bottom surface. Results for the blend nanofibers with a PAN to PVDF weight ratio of 1 to 1are shown in Figure 4(c). Initially, the water content of the bottom surface stays at zero because the water cannot pass easily through the mat. However, after a while, the water content increases although it remains much lower than the top layer. The blend nanofibers have a PAN-rich core and a PVDF-rich shell, and so the small amount of PAN in the shell enhances the wettability of the surface of the nanofibers. Thus the ability of the blend nanofibrous mat to transport water is better than that of pure PVDF. 


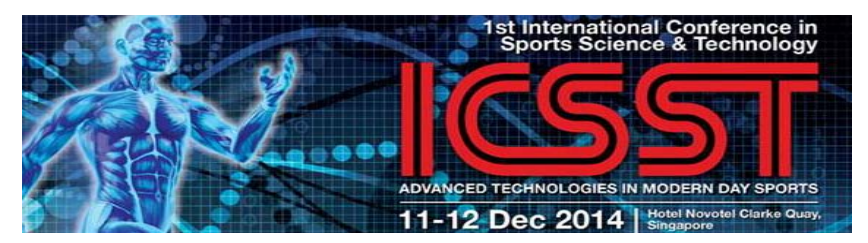

\section{Advanced Materials for \\ Sports Technology \\ www.icsst14.com}
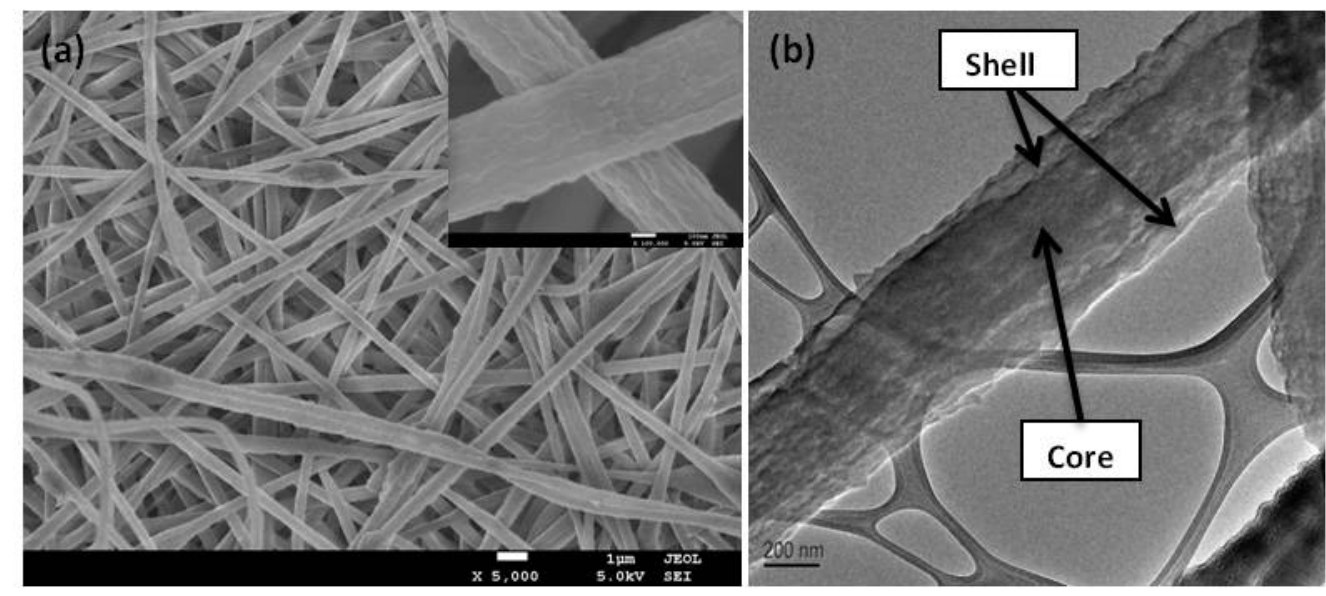

Figure1.SEM(a) and TEM(b) images of the blend (PAN: PVDF=1:1) nanofibers.

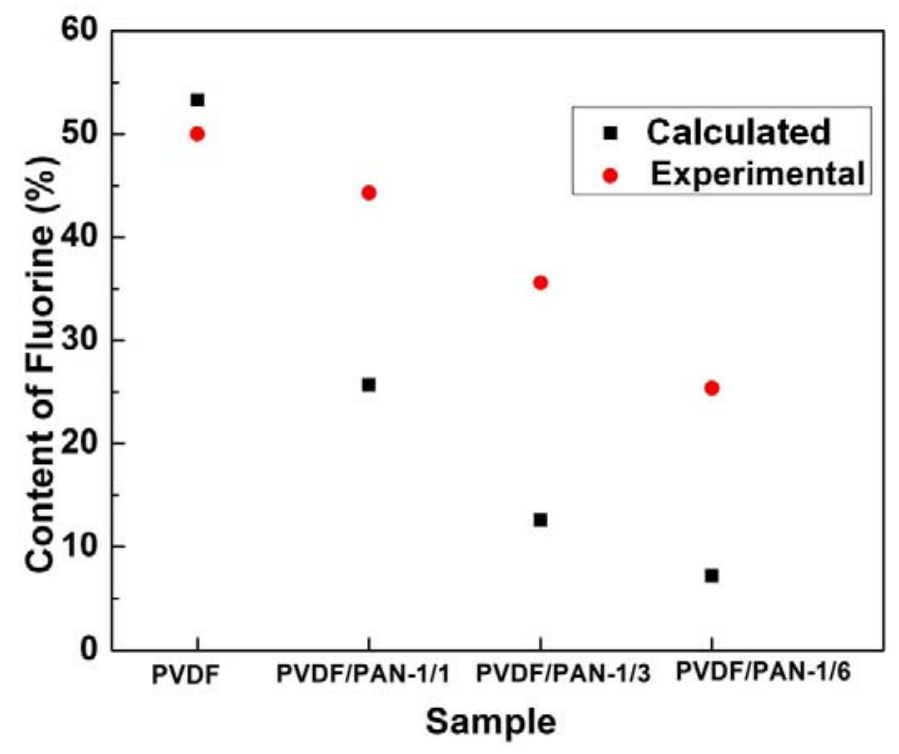

Figure2. Content of fluorine in PVDF nanofibers, blend nanofibers with PVDF/PAN ratios of 1/1, 1/3 and 1/6 measured by variable angle XPS and calculated values based on the feed ratio of the nanofibers 


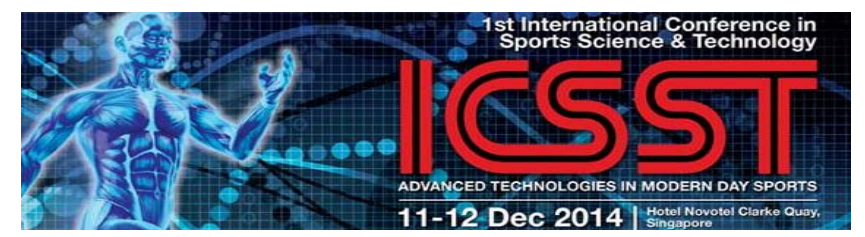

Advanced Materials for

Sports Technology

$\underline{\text { www.icsst14.com }}$

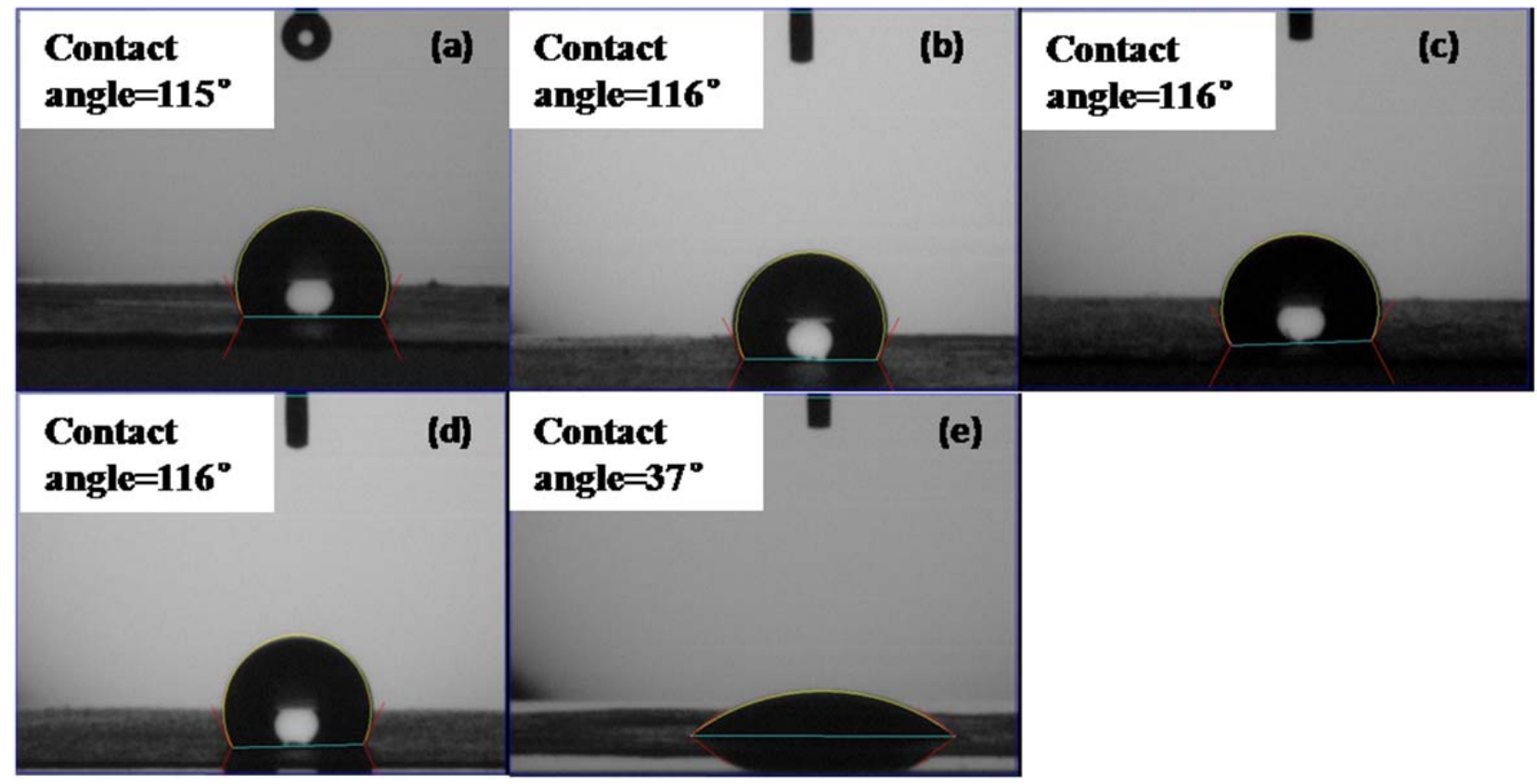

Figure3.Apparent contact angle of PVDF nanofibers (a), blend nanofibers with PVDF/PAN ratios of 1/1 (b), 1/3 (c), 1/6 (d) and PAN nanofibers(e).
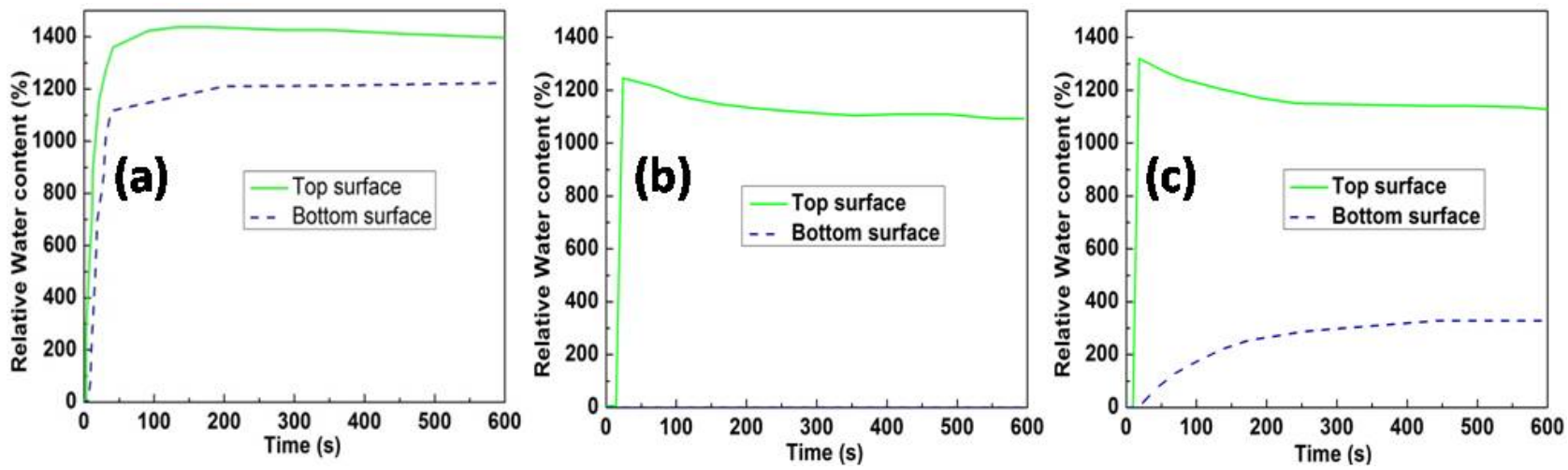

Figure4.The moisture management results of as-spunPAN (a), PVDF (b)and blend nanofibers with PVDF/PAN ratio

of $1 / 1(\mathrm{c})$.

\subsection{Base-treated CA nanofibersas outer layer}

Theoretically, a combination of a thin hydrophobic inner layer with a thick hydrophilic outer layer will pull the moisture outwards. The small thickness of the inner layer required is achievable by electrospinning. As discussed above, the PAN/PVDF blend electrospun mat is more wettable than 


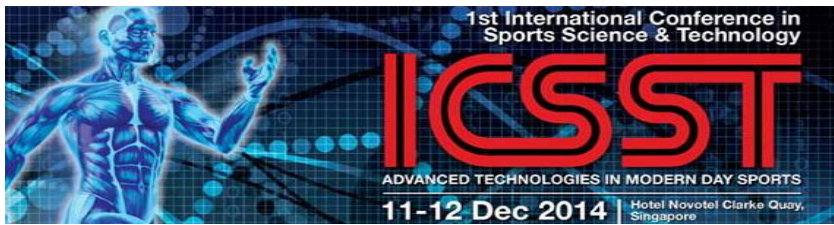

\section{Advanced Materials for Sports Technology \\ www.icsst14.com}

the neat PVDF mat and could allow water to pass through it. The thick hydrophilic outer layer, cellulose, which is the main component of traditional cotton fabric, was selected due to its high hydrophilicity and absorption. In this work, Cellulose nanofibers were fabricated by electrospinning of $\mathrm{CA}^{1819}$ followed by deacetylation to remove acetylgroups. ${ }^{20}$ In order to investigate the influence of deacetylation reaction time on the water transport property, cast CA films were soaked in $\mathrm{NaOH}$ aqueous solution for different times and their properties measured. According to the results of apparent contact angle and water absorption shown in Figure 5, the cast CA film becomes more hydrophilic with the increase of treatment time in $\mathrm{NaOH}$ aqueous solution. However, because the CA mats become fragile with too long treatment time, the CA nanofibrous mats were soaked in $\mathrm{NaOH}$ aqueous solution for 16 hours to partly convert $\mathrm{CA}$ into cellulose.

The CA nanofibrous mats soaked in $\mathrm{NaOH}$ aqueous solution for 4,8 and 16hours were tested by FTIR. The result is shown in Figure 6, from which it is seen that the characteristic peaks of CA becomes weaker while those of cellulose become more distinct with longer treatment time. Hence the effect of the reaction converting CA into cellulose is verified.

In addition, the apparent contact angle and moisture management results from a CA nanofibrous mat soaked in $\mathrm{NaOH}$ aqueous solution for 16 hours, as shown in Figure 7, reveal that the water can pass through the base-treated CA mats very quickly, indicating that base-treated CA is a suitable outer layer textile. The morphology of CA nanofibers and CA nanofibers soaked in $\mathrm{NaOH}$ aqueous solution for 16 hours are shown in Figure8, which shows that there is no obvious morphological variation after the treatment. 


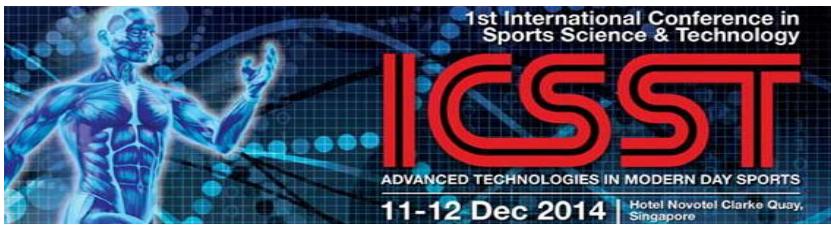

Advanced Materials for

Sports Technology

www.icsst14.com
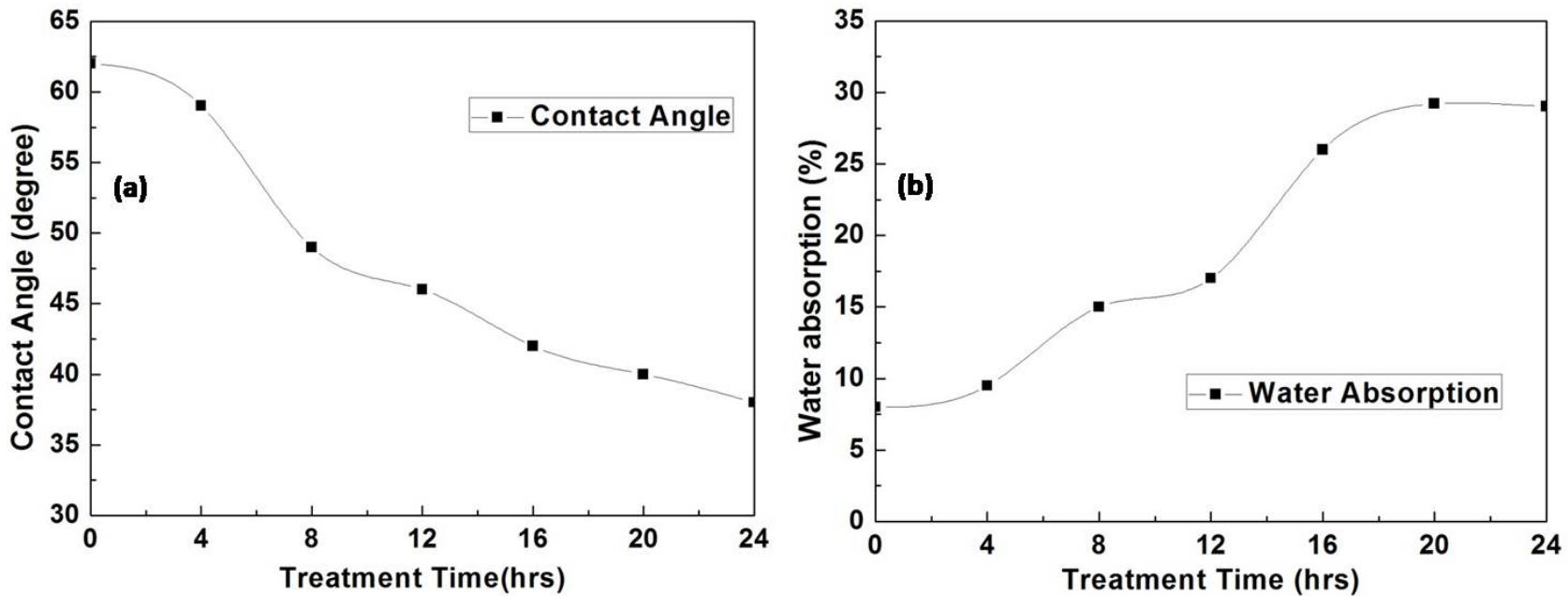

Figure5.Apparent contact angle and water absorption of CA mat and CA mat soaked in $\mathrm{NaOH}$ for 4, 8, 12, 16, 20 and $24 \mathrm{hrs}$

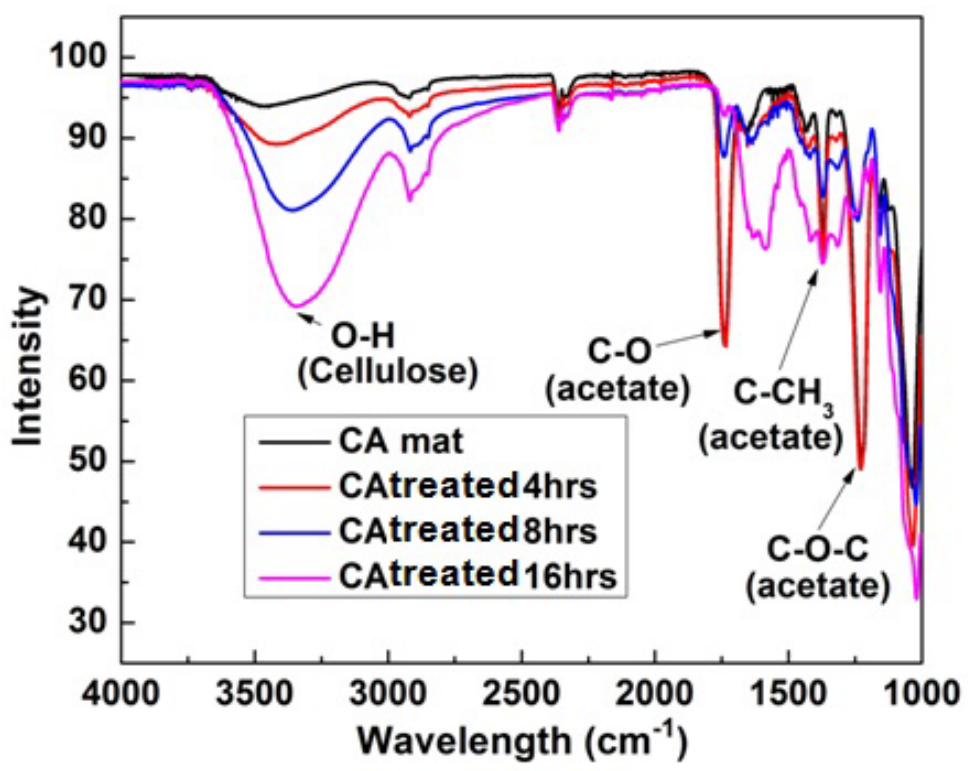

Figure6.FTIR spectra of CA mat and CA mat soaked in $\mathrm{NaOH}$ for 4,8 and $16 \mathrm{hrs}$ 


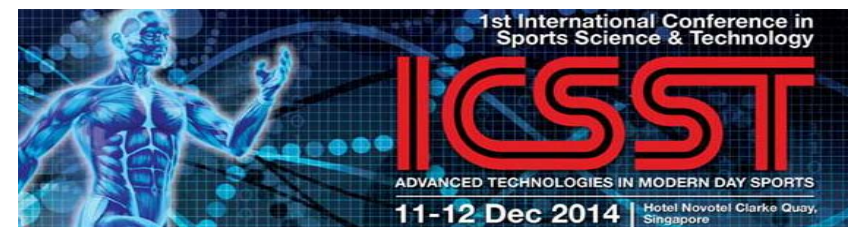

Advanced Materials for

Sports Technology

www.icsst14.com
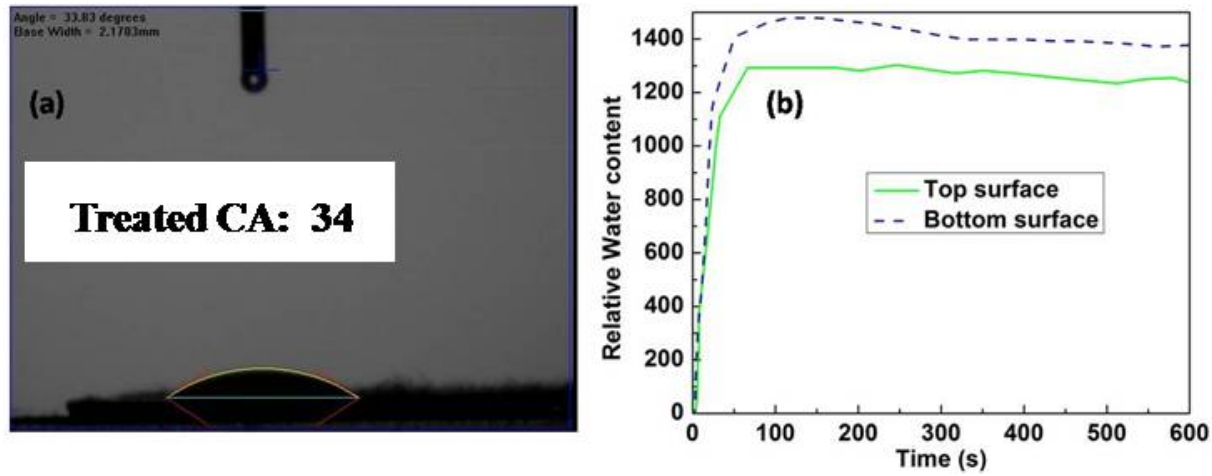

Figure7.Apparentcontact angle (a)and moisture management result (b) of CA mat soaked in $\mathrm{NaOH}$ for 16 hrs
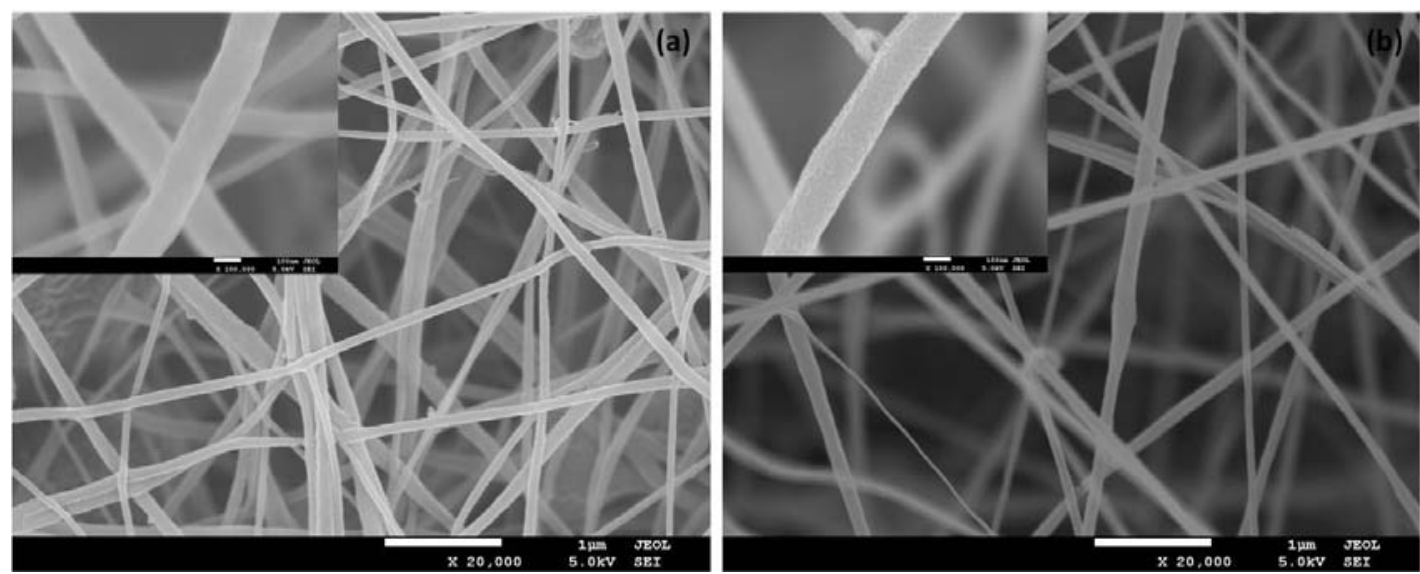

Figure8.SEM images of CA nanofibers and CA nanofibers soaked in $\mathrm{NaOH}$ for $16 \mathrm{hrs}$.

\subsection{Base-treated CA-PAN/PVDF dual-layer mats}

Base-treated CA-PAN/PVDF double-layer mats were prepared by electrospinning followed by going through a laminator to enhance the interlayer interactions. The schematic structure is shown in Figure 9a, which illustrates that moisture is effectively transported from the inner PAN/PVDF blend layer to the hydrophilic outer PAN layer due to push-pull effect. This is verified by the moisture management (MMT) results in Figure 9b.It is seen that the solid and dashed lines cross over after 300 seconds, showing that most water is transported from the top hydrophobic surface (inner surface) to the bottom hydrophilic surface (outer surface). 

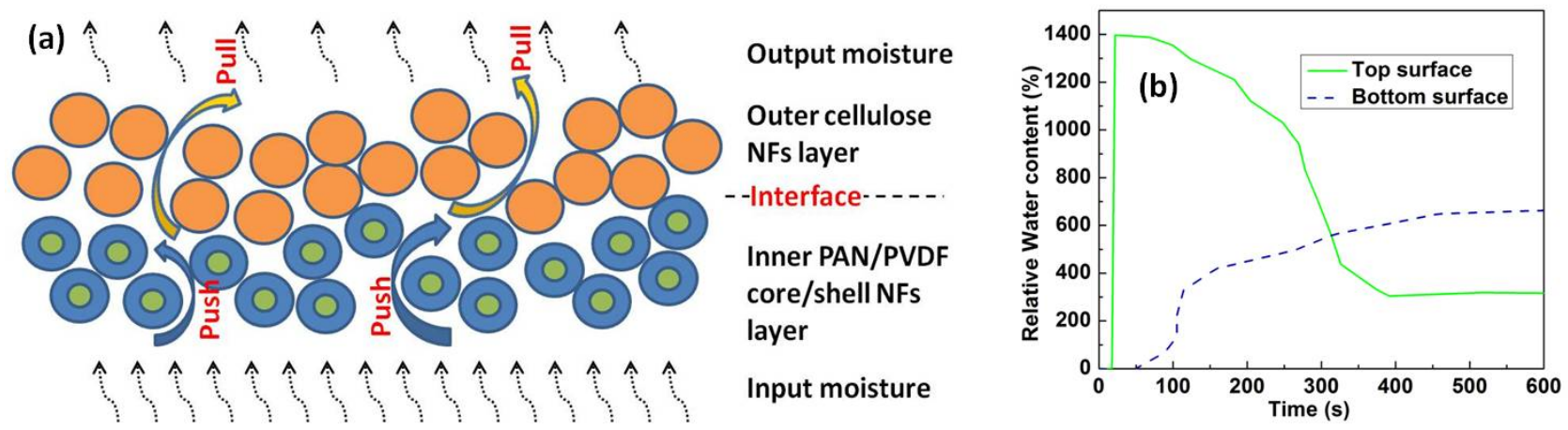

Figure9.Schematic diagram (a) and moisture management results of D-CA-PAN/PVDF mats(b).

\subsection{Friction coefficient of the inner layer}

Besides the moisture transport property, the friction coefficients of PAN, PVDF and PAN/PVDF blend nanofibrous mats were also measured and compared with traditional cotton fabric, using a Tribometer (CSM Instruments). The results are shown in Figure10. The nanofibrous mats all showed lower friction than the traditional cotton fabric, which is micron sized. Compared with the PAN mat, PVDF and PAN/PVDF mats show lower friction because of the fluorine component. To further verify the contribution of the fluorine component, friction coefficients of PAN, PVDF and PAN/PVDF blend cast films were also tested. The results, which are also shown inFigure10, reveal the same trend as the nanofibrous mats. Because the surfaces of the cast films are much rougher than those of the laminated electrospun mats, the friction coefficients of the cast films are higher than those of the electrospun mats. From the friction test results, it is seen that nanofibrous mats of PVDF and PAN/PVDF blend samples have similar friction coefficients due to the PVDF-rich shell structure of the blend mats. Hence the PAN/PVDF blend inner layer of the dual-layer structure would have a low friction coefficient with skin as well as better wettability compared with neat PVDF. 


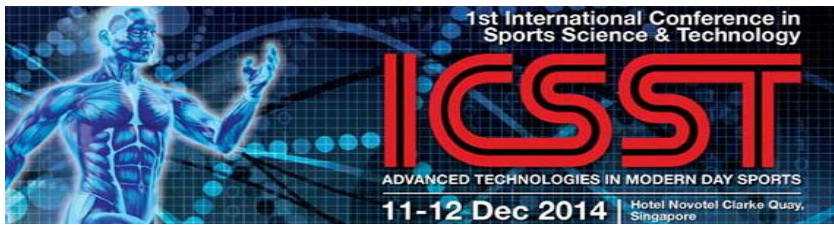

Advanced Materials for

Sports Technology

www.icsst14.com

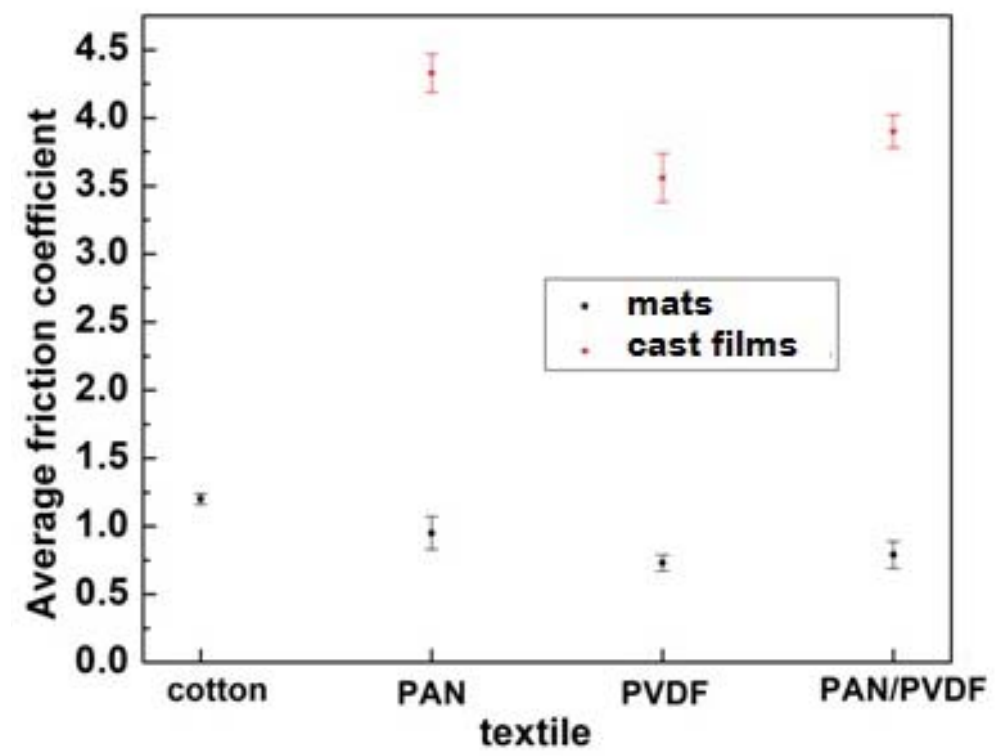

Figure10. The friction coefficients of cotton, PAN, PVDF and PAN/PVDF blend (at the ratio of 1/1) mats and cast films

\section{Conclusions}

In this work, electrospun dual-layer nanofibrous mats consisting of base-treated CA and core-shell PAN/PVDF nanofibers were designed and fabricated. The nanofibers with a PAN-rich core and a PVDF-rich shell were fabricated by single-spinneret electrospinning and used as the inner layer of the dual-layer mats, while thick base-treated CA nanofibrous mats were used as the outer hydrophilic layer. The core-located PAN and a small amount of PAN on the PAN/PVDF nanofiber surface ensure good moisture transport for the nanofibrous mats. The combination of a considerably hydrophobic PAN/PVDF inner layer and a highly hydrophilic CA outer layer induces a push-pull effect, effectively transporting moisture from the inner to the outer layer. Furthermore, the fluorine-rich PVDF shell of the inner layer touches the skin and may provide a lubricating effect to enhance comfortability. 


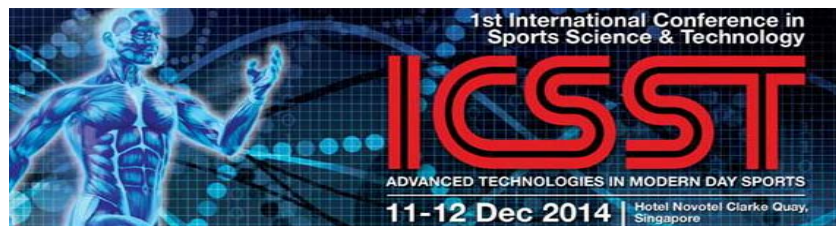

Advanced Materials for

Sports Technology

www.icsst14.com

\section{References}

1. Majumdar, A.; Mukhopadhyay, S.; Yadav, R., Thermal properties of knitted fabrics made from cotton and regenerated bamboo cellulosic fibres. International Journal of Thermal Sciences 2010, 49 (10), 2042-2048.

2. $\quad$ Markee, N. L.; Hatch, K. L.; Prato, H. H.; Zeronian, S. H.; Maibach, H. I., Effect of fiber type and fabric moisture content on the hydration state of human stratum corneum. Journal of Thermal Biology 1993, 18 (5), 421-427.

3. Tan, S.; Li, J.; Gao, G.; Li, H.; Zhang, Z., Synthesis of fluoropolymer containing tunable unsaturation by a controlled dehydrochlorination of P (VDF-co-CTFE) and its curing for high performance rubber applications. Journal of Materials Chemistry 2012, 22 (35), 18496-18504. 4. Bertaux, E.; Derler, S.; Rossi, R. M.; Zeng, X.; Koehl, L.; Ventenat, V., Textile, physiological, and sensorial parameters in sock comfort. Text. Res. J. 2010, 80 (17), 1803-1810. 5. Cazzaniga, S.; Lo Scocco, G.; Schincaglia, E.; Mercuri, S. R.; Chimenti, S.; Saraceno, R.; Naldi, L., Double-blind, within-patient, randomized, clinical trial comparing fluorine-synthetic fiber socks with standard cotton socks in improving plantar psoriasis. Journal of Dermatological Treatment 2014, 25 (1), 26-29.

6. Huang, J.; Li, S.; Ge, M.; Wang, L.; Xing, T.; Chen, G.; Liu, X.; Al-Deyab, S.; Zhang, K.Q.; Chen, T., Robust superhydrophobicTiO 2@ fabrics for UV shielding, self-cleaning and oilwater separation. Journal of Materials Chemistry A 2015, 3 (6), 2825-2832.

7. Rehan, M.; Mashaly, H. M.; Mowafi, S.; El-Kheir, A. A.; Emam, H. E., Multi-functional textile design using in-situ Ag NPs incorporation into natural fabric matrix. Dyes and Pigments 2015, 118, 9-17.

8. Yu, Z.-C.; Zhang, J.-F.; Lou, C.-W.; Lin, J.-H., Wicking behavior and antibacterial properties of multifunctional knitted fabrics made from metal commingled yarns. The Journal of The Textile Institute 2014, (ahead-of-print), 1-10.

9. $\quad$ Vasiljević, J.; Tomšič, B.; Jerman, I.; Orel, B.; Jakša, G.; Simončič, B., Novel multifunctional water-and oil-repellent, antibacterial, and flame-retardant cellulose fibres created by the sol-gel process. Cellulose 2014, 21 (4), 2611-2623.

10. Süpüren, G.; Oglakcioglu, N.; Ozdil, N.; Marmarali, A., Moisture management and thermal absorptivity properties of double-face knitted fabrics. Text. Res. J. 2011, 0040517511402122.

11. Agarwal, S.; Greiner, A.; Wendorff, J. H., Functional materials by electrospinning of polymers. Prog. Polym. Sci. 2013, 38 (6), 963-991.

12. Deitzel, J.; Kleinmeyer, J.; Harris, D. e. a.; Beck Tan, N., The effect of processing variables on the morphology of electrospun nanofibers and textiles. Polymer 2001, 42 (1), 261-272.

13. Huang, Z.-M.; Zhang, Y.-Z.; Kotaki, M.; Ramakrishna, S., A review on polymer nanofibers by electrospinning and their applications in nanocomposites. Composites science and technology 2003, 63 (15), 2223-2253.

14. Reneker, D. H.; Yarin, A. L., Electrospinning jets and polymer nanofibers. Polymer 2008, 49 (10), 2387-2425.

15. Dong, Y.; Kong, J.; Phua, S. L.; Zhao, C.; Thomas, N. L.; Lu, X., Tailoring Surface Hydrophilicity of Porous Electrospun Nanofibers to Enhance Capillary and Push-Pull Effects for Moisture Wicking. ACS applied materials \& interfaces 2014, 6 (16), 14087-14095.

16. Lu, W.; Liu, X.; Zhao, S.; Na, H.; Zhao, Y.; Yuan, X., Effect of benzyl triethylammonium chloride on microstructure of bicomponent polymeric fibers during electrospinning. Polymer Engineering \& Science 2012, 52 (8), 1661-1671. 


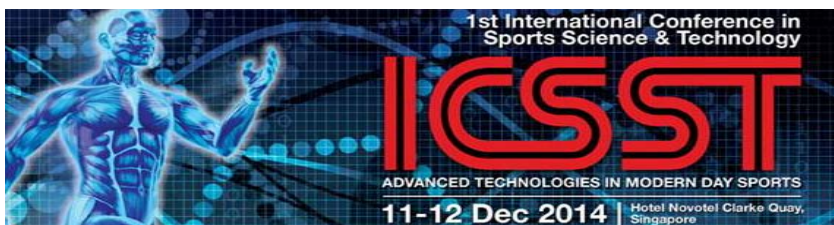

Advanced Materials for

Sports Technology

www.icsst14.com

17. Hu, J.; Li, Y.; Yeung, K.-W.; Wong, A. S.; Xu, W., Moisture management tester: A method to characterize fabric liquid moisture management properties. Textile Research Journal 2005, 75 (1), 57-62.

18. Konwarh, R.; Karak, N.; Misra, M., Electrospun cellulose acetate nanofibers: the present status and gamut of biotechnological applications. Biotechnology advances 2013, 31 (4), 421-437. 19. Anitha, S.; Brabu, B.; Thiruvadigal, D. J.; Gopalakrishnan, C.; Natarajan, T., Optical, bactericidal and water repellent properties of electrospun nano-composite membranes of cellulose acetate and $\mathrm{ZnO}$. Carbohydrate polymers 2013, 97 (2), 856-863.

20. Khatri, Z.; Wei, K.; Kim, B.-S.; Kim, I.-S., Effect of deacetylation on wicking behavior of co-electrospun cellulose acetate/polyvinyl alcohol nanofibers blend. Carbohydrate Polymers 2012, 87 (3), 2183-2188. 